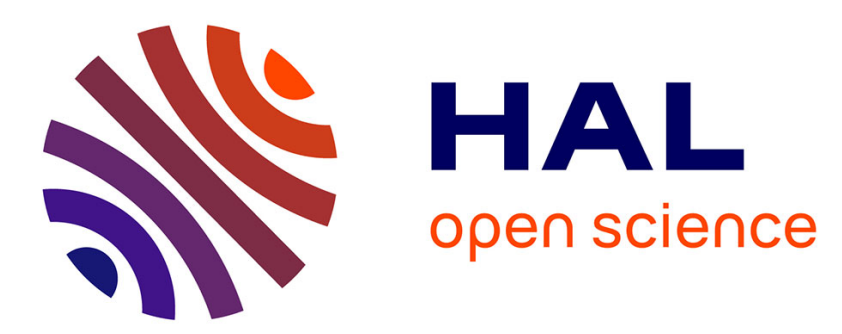

\title{
On the equilibrium equation for a generalized biological membrane energy by using a shape optimization approach
}

Aymen Laadhari, Chaouqi Misbah, Pierre Saramito

\section{- To cite this version:}

Aymen Laadhari, Chaouqi Misbah, Pierre Saramito. On the equilibrium equation for a generalized biological membrane energy by using a shape optimization approach. Physica D: Nonlinear Phenomena, 2010, 239 (16), pp.1567-1572. 10.1016/j.physd.2010.04.001 . hal-00388733

\section{HAL Id: hal-00388733 \\ https://hal.science/hal-00388733}

Submitted on 27 May 2009

HAL is a multi-disciplinary open access archive for the deposit and dissemination of scientific research documents, whether they are published or not. The documents may come from teaching and research institutions in France or abroad, or from public or private research centers.
L'archive ouverte pluridisciplinaire HAL, est destinée au dépôt et à la diffusion de documents scientifiques de niveau recherche, publiés ou non, émanant des établissements d'enseignement et de recherche français ou étrangers, des laboratoires publics ou privés. 


\title{
On the equilibrium equation for a generalized biological membrane energy by using a shape optimization approach
}

\author{
A. Laadhari ${ }^{\mathrm{a}, \mathrm{b}}$, C. Misbah ${ }^{*, a}$, P. Saramito ${ }^{\mathrm{b}}$ \\ ${ }^{a}$ Laboratoire de Spectrométrie Physique, 140 avenue de la physique, Université Joseph \\ Fourier, and CNRS, 38402, Saint Martin d'Hères, France \\ ${ }^{b}$ Laboratoire Jean Kuntzmann, Université Joseph Fourier and CNRS, B. P. 53, 38041 \\ Grenoble Cedex 9, France
}

\begin{abstract}
A mechanical equilibrium equation of a vesicle membrane under a generalized elastic bending energy is obtained in this paper. Moreover, the derivation of this equilibrium equation bases on some shape optimization tools. This approach is new and more concise than the tensorial tools previously used for this problem.
\end{abstract}

Key words: Vesicle shape, red cell membrane, Helfrich energy, shape optimization

PACS: ...

\section{Introduction}

Phospholipid membranes are abundant in biology. They represent the major component of the cytoplasmic membrane of real cells. They are also present within the cell cytoplasm (e.g. Golgi apparatus, a complex assembly of phospholipid layers which serve to form small vesicles for protein transport). Phospholipid membranes are also used in many industrial application (e.g. giant liposome emulsions for cosmetics). Pure phospholipid vesicles (a closed membrane suspended in an aqueous solution) constitute an attractive model system in order to describe mechanical and viscoelastic behaviors of many cells, like red blood cells. They are also regraded as promising drug carriers for a delivery at specific sites in the organisms. This explains the increasing interest for biological membranes from various communities ranging from biology to applied mathematics. This contribution is concerned with a certain aspect of mathematical modeling of vesicles, or more generally of phospholipid membranes.

\footnotetext{
${ }^{*}$ Corresponding author

Email addresses: Aymen.Laadhari@imag.fr (A. Laadhari), cmisbah@ujf-grenoble.fr (C. Misbah), Pierre.Saramito@imag.fr (P. Saramito)
} 
Vesicles are formed by amphiphilic molecules self-assembled in water to build bilayers, in a certain range of concentration and temperature. Many recent experimental and theoretical studies focus on the configuration and equilibrium shape of elastic biomembranes under flow (see [1]) with the aim of understanding dynamics and rheology.

At room, as well as at the physiological temperature, the membrane is fluid (a two dimensional incompressible fluid). Due to incompressibility, the main mode of deformation of a vesicle is bending. A basic ingredient for biomembranes is thus bending energy. Helfrich [2] introduced a model in which the cost in bending energy is given by

$$
\frac{\alpha}{2} \int_{\Gamma}\left(H-H_{0}\right)^{2} \mathrm{~d} s+\frac{\beta}{2} \int_{\Gamma} K \mathrm{~d} s,
$$

where $H=\kappa_{1}+\kappa_{2}$ is the mean curvature of the membrane surface, with $\kappa_{1}$ and $\kappa_{2}$ are the principle curvatures, $K=\kappa_{1} \kappa_{2}$ is the Gauss curvature and $H_{0}$ represents the spontaneous curvature that describe the asymmetry effect of the membrane or its environment. The membrane surface is denoted by $\Gamma$ while $\Omega$ represents the inside volume of the vesicle, such that $\Gamma=\partial \Omega$. The integrals are performed along the membrane surface where $\mathrm{d} s$ denotes a surface area, while, in this paper, $\mathrm{d} x$ will represent a volume element. The constants $\alpha$ and $\beta$ have the dimension of an energy and represent the bending rigidity and the Gaussian rigidity, respectively. The second term in the Helfrich model is a topological invariant by the virtue of the Gauss-Bonnet theorem that says if one is not interested in change of topology, then this contribution is a constant and can be ignored. We shall disregard in the energy the contribution coming from Gauss curvature, since we do not account for topological changes.

The equilibrium shape of vesicle membranes is determined by minimizing the bending energy subject to two constraints: fixed volume (incompressible enclosed fluid) and fixed area (inextensible membrane). It is a shape optimization problem that writes in the saddle point formulation as follows: find $(\Omega, \zeta, p)$ such that

$$
\inf _{\Omega \subset R^{3}} \sup _{\zeta, p \in R} L(\Omega,(\zeta, p))
$$

where $L$ is the Lagrangian

$$
L(\Omega,(\zeta, p))=\int_{\Gamma} f(H) \mathrm{d} s+\zeta\left(\int_{\Gamma} \mathrm{d} s-A_{0}\right)+p\left(\int_{\Omega} \mathrm{d} x-V_{0}\right) .
$$

The scalars $\zeta$ and $p$ are Lagrange multipliers, and can be viewed as an effective surface tension and pressure difference, respectively. They enforce constant area $A_{0}$ and constant volume $V_{0}$ of the vesicle, respectively. The Helfrich energy has been generalized by introducing $f$, an arbitrary smooth function defined in $R$. Notice that the classical case corresponds to the choice $f(H)=\frac{\alpha}{2}\left(H-H_{0}\right)^{2}$.

The notion of saddle point can be intuitively understood as follows. We would like the energy to be minimal (or $L$ to be minimal with respect to shape variation) and that at the same time $L$ should behave with respect to $\zeta$ and $p$ 
in a such a way to be maximum, so that to enforce constant volume and area (i.e. to suppress the terms proportional to $\zeta$ and $p$ in $L$ ).

Since $L$ is differentiable, any saddle-point $(\Omega,(\zeta, p))$ of $L$ satisfies three conditions:

$$
\frac{\partial L}{\partial \Omega}(\Omega,(\zeta, p))=0, \quad \frac{\partial L}{\partial \zeta}(\Omega,(\zeta, p))=0 \quad \text { and } \quad \frac{\partial L}{\partial p}(\Omega,(\zeta, p))=0 .
$$

The two last conditions leads directly to the area and volume constraints, respectively. The aim of this paper is to show that the first condition, that involves the shape derivative $\partial / \partial \Omega$, leads to:

$$
p+\zeta H+f(H) H+\left(2 K-H^{2}\right) f^{\prime}(H)-\Delta_{s}\left(f^{\prime}(H)\right)=0 .
$$

Notice that the choice $f(H)=\frac{\alpha}{2}\left(H-H_{0}\right)^{2}$ leads to the classical equilibrium condition:

$$
p+\zeta H+\alpha\left(\frac{1}{2}\left(H-H_{0}\right)\left[4 K-H\left(H+H_{0}\right)\right]-\Delta_{s} H\right)=0 .
$$

where $\Delta_{s}$ is the surface Laplacian (known also as the Laplace-Beltrami operator), and will be defined explicity in this paper. The result (3) was first derived in [2], relation (31). Notice that these authors used the same notation $H$ for $-\left(\kappa_{1}+\kappa_{2}\right) / 2$, i.e. minus half of the present definition of the mean curvature $H=\kappa_{1}+\kappa_{2}$. In that paper use of several concepts of differential geometry were evoked (first and second fundamental forms) in order to arrive to the final result.

The main objective of this paper is to provide a derivation which is concise and self-contained. Our derivation uses quite simple and classical notions. While we will, in passing, quote some known expressions and Lemma in the mathematical literature, we shall provide their direct derivation here. In addition, our derivation can be made general without specifying the functional expression $f(H)$. We shall discuss in the conclusion when generalized functionals can be expected to arise.

The paper is organized as follow: the second section introduces some notations and preliminary results while the third one aim at obtaining the equilibrium condition for the generalized expression (1) of the Helfrich energy. The paper is completed by a mathematical appendix.

\section{Notations and preliminary results}

All surface operators used in this paper are defined here. Let $\mathbf{n}$ denotes the unit outward normal vector to the shape $\Omega$. Let $f$ be any scalar function and $\mathbf{v}$ be any vector field. The surface gradient, the surface divergence and the Laplace-Beltrami operator are respectively expressed by:

$$
\begin{aligned}
\nabla_{s} f & =(\mathbf{I}-\mathbf{n} \otimes \mathbf{n}) \nabla f=\nabla f-(\mathbf{n} \cdot \nabla f) \mathbf{n}, \\
\nabla_{s} \cdot \mathbf{v} & =(\mathbf{I}-\mathbf{n} \otimes \mathbf{n}): \nabla \mathbf{v}=\nabla \cdot \mathbf{v}-((\nabla \mathbf{v}) \cdot \mathbf{n}) \cdot \mathbf{n}, \\
\Delta_{s} f & =\nabla_{s} \cdot\left(\nabla_{s} f\right) .
\end{aligned}
$$




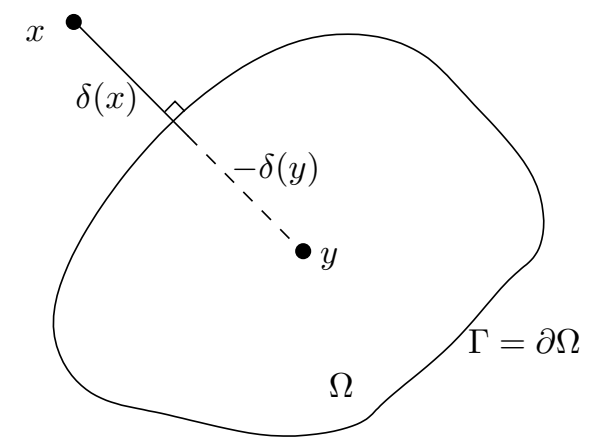

Figure 1: Schematic view of the geometry. $\delta$, the distance function, is positive for $x$ outside $\Omega$ and negative inside. This is the signed distance.

The boundary $\Gamma$ is then parameterized by a level set function $\delta$, defined, for all $x \in R^{3}$, as a signed distance (see Figure (1)):

$$
\delta(x)=\left\{\begin{aligned}
\inf _{y \in \Gamma}|y-x| & \text { when } x \notin \Omega, \\
-\inf _{y \in \Gamma}|y-x| & \text { otherwise. }
\end{aligned}\right.
$$

Then, the normal expresses as a gradient: $\mathbf{n}=\nabla \delta$ (since $|\nabla \delta|=1$.

Let us now express the mean and the Gauss curvatures. Let $A=\nabla_{s} \mathbf{n}$. Its characteristic polynom writes:

$$
\mathcal{P}_{A}(\lambda)=\operatorname{det}(A-\lambda \mathbf{I})=-\lambda^{3}+I_{1} \lambda^{2}-I_{2} \lambda+\operatorname{det}(A),
$$

where $I_{1}=A_{i i}$ and $I_{2}=\left(A_{i i} A_{j j}-A_{i j} A_{j i}\right) / 2$ (repeated indices convention is used), are two invariants; see below.

Let us first show that $A=\nabla_{s} \mathbf{n}=\nabla \mathbf{n}$. From the definition (4) of $\nabla_{s}$, it is equivalent to show that $\mathbf{n} . \nabla \mathbf{n}=0$. On the one hand, since $|\mathbf{n}|^{2}=1$, we have $\nabla(\mathbf{n} . \mathbf{n})=0$. On the other hand, by expansion we have $\nabla(\mathbf{n} . \mathbf{n})=$ $2(\mathbf{n} . \nabla) \mathbf{n}+2 \mathbf{n} \wedge \operatorname{rot}(\mathbf{n})$. Then $(\mathbf{n} . \nabla) \mathbf{n}=-2 \mathbf{n} \wedge \operatorname{rot}(\mathbf{n})$. Next, since $\mathbf{n}=\nabla \delta$, we have $\operatorname{rot}(\mathbf{n})=\operatorname{rot}(\nabla \delta)=0$. Finally $\mathbf{n} . \nabla \mathbf{n}=0$ and then $A=\nabla \mathbf{n}$.

Moreover, from definition of $A$, it is also the Hessian of the level set function: $A=(\nabla \otimes \nabla) \delta$. Thus $A$ is symmetric and admits three real eigenvalues. Since $\mathbf{n} \cdot \nabla \mathbf{n}=\mathbf{n} \cdot A=0, A$ has a zero eigenvalue, associated to the eigenvector $\mathbf{n}$. Let us denote $\kappa_{1}, \kappa_{2}$ the two others eigenvalues of $A$. There exists an orthonormal eigenvector system $\left(\mathbf{e}_{1}, \mathbf{e}_{2}, \mathbf{n}\right)$ associated to $\left(\kappa_{1}, \kappa_{2}, 0\right)$ such that $A=\kappa_{1} \mathbf{e}_{1} \otimes \mathbf{e}_{1}+\kappa_{2} \mathbf{e}_{2} \otimes \mathbf{e}_{2}$. By definition [3, p. 47], the values $\kappa_{1}$ and $\kappa_{2}$ are called the principle curvatures and the vectors $\mathbf{e}_{1}$ and $\mathbf{e}_{2}$, the principle directions of curvature. The mean curvature and the Gauss curvature are, by definition $H=\kappa_{1}+\kappa_{2}$ and $K=\kappa_{1} \kappa_{2}$, respectively. Notice that as coefficients of the characteristic polynom, they are invariant by any change of base, and thus in the eigensystem we find: $I_{1}=H$ and $I_{2}=K$. Going back to the definition of 
$I_{1}$ and $I_{2}$ in terms of $A=\nabla \mathbf{n}$ leads to the following expression of the mean and the Gauss curvatures:

$$
H=\nabla_{s} \cdot \mathbf{n}=\nabla \cdot \mathbf{n} \text { and } 2 K=H^{2}-\nabla \mathbf{n}: \nabla \mathbf{n}^{T} .
$$

Notice that the use of the level set function $\delta$ enables us to extend the quantities $\mathbf{n}, H$ and $K$ in the whole $R^{3}$ space while their original definitions was introduced only on the surface $\Gamma$.

\section{Obtaining the equilibrium equation}

Let us denote by $E(\Omega), A(\Omega, \zeta)$ and $V(\Omega, p)$ the first, second and third terms of the right-hand side in (1), respectively. From Lemma A.1 derived in appendix $\mathrm{A}$, we get:

$$
\frac{\partial V}{\partial \Omega}(\Omega, p)(\mathbf{u})=p \int_{\Gamma} \mathbf{u} \cdot \mathbf{n} \mathrm{d} s .
$$

Again, from Lemma A.1:

$$
\frac{\partial A}{\partial \Omega}(\Omega, \zeta)(\mathbf{u})=\zeta \int_{\Gamma} H \mathbf{u} . \mathbf{n} \mathrm{d} s .
$$

The rest of the paragraph deals with the $E(\Omega)$ term. From Lemma A.2 derived in the appendix, we obtain:

$$
\frac{\partial E}{\partial \Omega}(\Omega)(\mathbf{u})=\int_{\Gamma} \frac{\partial f(H)}{\partial \Omega}(\Omega)(\mathbf{u}) \mathrm{d} s+\int_{\Gamma}\left(f(H)(\Omega) H+\frac{\partial(f(H))(\Omega)}{\partial \mathbf{n}}\right) \mathbf{u . n} \mathrm{d} s,
$$

where $\partial / \partial \mathbf{n}=\mathbf{n} . \nabla$ denotes the directional (or normal) derivative. The normal $\mathbf{n}$, which also depends on the shape $\Omega$ is differentiable and its shape derivative in any direction $\mathbf{u}$ writes $[4]: \frac{\partial \mathbf{n}}{\partial \Omega}(\Omega)(\mathbf{u})=-\nabla_{s}(\mathbf{u} . \mathbf{n})$. This expression can be derived by considering the elementary local displacement $\mathbf{u}$ of the interface $\Gamma$. In such a case the level set function $\delta$, that has the material derivative equal to zero, satisfies the transport equation in time $t$ with the velocity $\mathbf{v}$

$$
\frac{d \delta}{d t}+\nabla \delta \cdot \mathbf{v}=\mathbf{0}
$$

this leads to :

$$
d \delta=-\nabla \delta . \mathbf{u}
$$

where $d \delta$ represents an elementary local displacement of the shape.

The shape derivative of the normal is given by the Fréchet differentiation of $\frac{\nabla \delta(x)}{|\nabla \delta(x)|}$, in the direction $d \delta$, since the propriety $|\nabla \delta(x)|=1$ for all position $x$ in $R^{3}$, is not preserved if the displacement $d \delta$ is applied. Consequently, using (11), the shape derivative of $\mathbf{n}$ is given by

$$
\lim _{\varepsilon \rightarrow 0} \frac{\mathbf{n}(\delta+\varepsilon \mathrm{d} \delta)-\mathbf{n}(\delta)}{\varepsilon}=\frac{\nabla(-\mathbf{u} \cdot \nabla \delta)}{|\nabla \delta|}-\frac{[\nabla(-\mathbf{u} \cdot \nabla \delta) \cdot \nabla \delta] \nabla \delta}{|\nabla \delta|^{3}}=-\nabla_{s}(\mathbf{u} \cdot \mathbf{n})
$$


Noting that, by considering the elementary local displacement $d \delta$, we have $|\nabla \delta(x)| \rightarrow 1$ when $\varepsilon \rightarrow 0$. This result can be shown otherwise (see [5, eq. A7]). From $H=\nabla . \mathbf{n}$, we obtain $\frac{\partial H}{\partial \Omega}(\Omega)(\mathbf{u})=-\nabla \cdot\left[\nabla_{s}(\mathbf{u} . \mathbf{n}(\Omega))\right]$ and then:

$$
\frac{\partial f(H)}{\partial \Omega}(\Omega)(\mathbf{u})=f^{\prime}(H) \frac{\partial H}{\partial \Omega}(\Omega)(\mathbf{u})=-f^{\prime}(\nabla \cdot \mathbf{n}) \nabla \cdot\left[\nabla_{s}(\mathbf{u} \cdot \mathbf{n})\right] .
$$

Next, (10) leads to:

$\frac{\partial E}{\partial \Omega}(\Omega)(\mathbf{u})=\int_{\Gamma} f(H)(\Omega) H \mathbf{u . n} \mathrm{d} s+\int_{\Gamma} f^{\prime}(H) \frac{\partial H}{\partial \mathbf{n}} \mathbf{u . n} \mathrm{d} s-\int_{\Gamma} f^{\prime}(H) \nabla \cdot\left[\nabla_{s}(\mathbf{u} . \mathbf{n})\right] \mathrm{d} s$.

Let us denote by $T$ the last term of the right-hand side. The classical Green formula leads to:

$$
T=-\int_{\Gamma} f^{\prime}(H) \nabla \cdot\left(\nabla_{s}(\mathbf{u} \cdot \mathbf{n})\right) \mathrm{d} s=\int_{\Gamma} \nabla_{s}(\mathbf{u} \cdot \mathbf{n}) \cdot \nabla\left(f^{\prime}(H)\right) \mathrm{d} s .
$$

Recall that, for any scalar function $g$ and any vector field $\mathbf{v}$ defined over the closed surface $\Gamma$, we have the following identity (see appendix C):

$$
\int_{\Gamma} \nabla_{s} g \cdot \mathbf{v} \mathrm{d} s=-\int_{\Gamma} g \nabla_{s} \cdot \mathbf{v} \mathrm{d} s+\int_{\Gamma} g \mathbf{v} \cdot \mathbf{n} H \mathrm{~d} s .
$$

With $g=\mathbf{u} . \mathbf{n}$ and $\mathbf{v}=\nabla\left(f^{\prime}(H)\right)$, we get:

$$
T=-\int_{\Gamma} \nabla_{s} \cdot\left(\nabla\left(f^{\prime}(H)\right)\right) \text { u.n } \mathrm{d} s+\int_{\Gamma} \frac{\partial\left(f^{\prime}(H)\right)}{\partial \mathbf{n}} H \mathbf{u} . \mathbf{n} \mathrm{d} s .
$$

Then (13) becomes:

$\frac{\partial E}{\partial \Omega}(\Omega)(\mathbf{u})=\int_{\Gamma}\left(f(H) H+f^{\prime}(H) \frac{\partial H}{\partial \mathbf{n}}+\frac{\partial\left(f^{\prime}(H)\right)}{\partial \mathbf{n}} H-\nabla_{s} \cdot\left[\nabla\left(f^{\prime}(H)\right)\right]\right) \mathbf{u} \cdot \mathbf{n} \mathrm{d} s$.

This completes our derivation, in principle. However, some useful simplifications can be made, as shown below.

- Simplification 1. Using the summation of repeated indices convention, we have:

$\frac{\partial H}{\partial \mathbf{n}}=\mathbf{n} . \nabla(\nabla . \mathbf{n})=\mathbf{n}_{i} \partial_{i} \partial_{j} \mathbf{n}_{j}=\partial_{j}\left(\mathbf{n}_{i} \partial_{i} \mathbf{n}_{j}\right)-\partial_{i} \mathbf{n}_{j} \partial_{j} \mathbf{n}_{i}=\nabla \cdot((\mathbf{n} . \nabla) \mathbf{n})-\nabla \mathbf{n}: \nabla \mathbf{n}^{T}$.

Next, using expression (7) of the Gauss curvature $K$, we obtain:

$$
\frac{\partial H}{\partial \mathbf{n}}=2 K-H^{2}+\nabla \cdot[(\mathbf{n} \cdot \nabla) \mathbf{n}]
$$

Finally, since $\mathbf{n} . \nabla \mathbf{n}=0$ (see section 2) we get:

$$
\frac{\partial H}{\partial \mathbf{n}}=2 K-H^{2} .
$$


- Simplification 2. From (4) we have:

$$
\begin{aligned}
-\nabla_{s} \cdot\left\{\nabla\left(f^{\prime}(H)\right)\right\} & =-\nabla_{s} \cdot\left\{\nabla_{s}\left(f^{\prime}(H)\right)+\left(\mathbf{n} \cdot \nabla\left(f^{\prime}(H)\right)\right) \mathbf{n}\right\} \\
& =-\Delta_{s}\left(f^{\prime}(H)\right)-\left(\mathbf{n} \cdot \nabla_{s}\right)\left\{\mathbf{n} \cdot \nabla\left(f^{\prime}(H)\right)\right\}-(\mathbf{n} \cdot \nabla)\left(f^{\prime}(H)\right)(\nabla \cdot \mathbf{n}) .
\end{aligned}
$$

Notice that, from definition (4) of $\nabla_{s}$, we have $\mathbf{n} \cdot \nabla_{s}=0$ and then:

$$
-\nabla_{s} \cdot\left(\nabla\left(f^{\prime}(H)\right)\right)=-\Delta_{s}\left(f^{\prime}(H)\right)-\frac{\partial\left(f^{\prime}(H)\right)}{\partial \mathbf{n}} H .
$$

Using the two simplifications (16) and (17), relation (15) becomes:

$$
\frac{\partial E}{\partial \Omega}(\Omega)(\mathbf{u})=\int_{\Gamma}\left\{f(H) H+f^{\prime}(H)\left(2 K-H^{2}\right)-\Delta_{s}\left(f^{\prime}(H)\right)\right\} \mathbf{u . n} \mathrm{d} s .
$$

Finally, using relation (8),(9), the previous relation leads to:

$$
\frac{\partial L}{\partial \Omega}(\Omega,(\zeta, p))(\mathbf{u})=\int_{\Gamma}\left\{f(H) H+f^{\prime}(H)\left(2 K-H^{2}\right)-\Delta_{s}\left(f^{\prime}(H)\right)+p+\zeta H\right\} \mathbf{u} . \mathbf{n} \mathrm{d} s .
$$

This last expression leads directly to the equilibrium condition (2).

\section{Conclusion}

We have provided a new and self-contained derivation of the force for a biological membrane. We have extended the derivation to a generalized expression of the functional $f(H)$. The Helfrich energy can be motivated by assuming that the stretching energy is of harmonic type (the energy is proportional to the square of change of distance from a certain configuration). In more complex situations, like red blood cells for example, this assumption is not obvious, in that a nonlinear constitutive law may constitute a better approximation. The resulting bending energy should thus lead to a different nonlinear function of mean curvature. While this is, intuitively, a quite plausible situation in real systems, we are not aware of any work who has dealt with this case yet.

Another, perhaps more frequent, situation is that where macromolecules (like proteins) interact with a membrane [6]. The Helfrich energy $\alpha / 2 \int_{\Gamma}(H-$ $\left.H_{0}\right)^{2}$ should be supplemented with a term of the form $\int_{\Gamma} F(c)$ where $F$ is certain function of protein concentration. In addition, $H_{0}$ (the spontaneous curvature) is generally a $c$-dependent function (see [6]). Minimisation of the full energy with respect to $c$ yields a relation

$$
F^{\prime}(c)+\alpha\left(H-H_{0}\right) H_{0}^{\prime}(c)=0
$$

Solving for $c$ yields, generically a nonlinear relation between $c$ and $H$, and reporting the result into the original energy amounts to minimizing a generalized functional of the form $\int_{\Gamma} f(H)$, that is an expression of the form dealt with in this paper. 


\section{References}

[1] C. Misbah, Vacillating breathing and tumbling of vesicles under shear flow, Phys. Rev. Lett. 96 (2006) 028104.

[2] O.-Y. Zhong-can, W. Helfrich, Bending energy of vesicle membranes: general expressions for the first, second and third variation of the shape energy and applications to spheres and cylinders, Phys. Rev. A 39 (10) (1989) 52805288.

[3] D. A. Edwards, H. Brenner, D. T. Wasan, Interfacial transport processes and rheology, Butterworth-Heinemann, 1961.

[4] J. Simon, Differentiation with respect to the domain in boundary value problems, J. Num. Funct. Anal. Optim. 2 (7-8) (1980) 649-687.

[5] T. Biben, K. Kassner, C. Misbah, Phase-field approach to three-dimensional vesicle dynamics, Phys. Rev. E 72 (2005) 041921.

[6] F. Divet, G. Danker, C. Misbah, Fluctuations and instability of a biological membrane induced by interaction with macromolecules, Phys. Rev. E 73 (2002) 041901-041914.

[7] G. Allaire, F. Jouve, Minimum stress optimal design with the level set method, International Journal of Boundary Elements and Applications (accepted).

[8] F. Murat, J. Simon, Quelques résultats sur le contrôle par un domaine géométrique, Tech. Rep. LA 189, Lab. d'Analyse Numérique, Univ. Paris VI (1974).

[9] B. Kaoui, G. H. Ristow, I. Cantat, C. Misbah, W. Zimmermann, Lateral migration of a two-dimensional vesicle in unbounded poiseuille flow, Phys. Rev. E 77 (2008) 021903.

\section{A. Some shape optimization tools}

The notion and basic tools of shape derivative $[7,4,8]$ is recalled in this appendix. Starting from a smooth reference open set $\Omega$, with boundary $\Gamma=\partial \Omega$, we consider domains of the type $\Omega_{\mathbf{u}}=(\mathbf{I}+\mathbf{u})(\Omega)$ with $\mathbf{I}$ denotes the identity in $R^{3}$ and $\mathbf{u}$ is any sufficiently regular vector field.

Definition A.1. The shape derivative of $J(\Omega)$ versus $\Omega$ is defined as the Fréchet derivative at $\mathbf{u}=0$ of $\mathbf{u} \rightarrow J((\mathbf{I}+\mathbf{u})(\Omega))$, i.e.

$$
J((\mathbf{I}+\mathbf{u})(\Omega))=J(\Omega)+\frac{\partial J}{\partial \Omega}(\Omega)(\mathbf{u})+o(\mathbf{u}) \text { with } \lim _{\mathbf{u} \rightarrow 0} \frac{\|o(\mathbf{u})\|}{\|\mathbf{u}\|}=0,
$$

where $J^{\prime}(\Omega)(\mathbf{u})$ is linear and continuous with respect to $\mathbf{u}$ and where $\|\cdot\|$ denotes the usual Euclidian norm in $R^{3}$. 
Lemma A.1. Considering a smooth bounded open set $\Omega \in R^{3}$ and $f(x)$ a smooth function defined in $R^{3}$. Define

$$
J_{v o l}(\Omega)=\int_{\Omega} f(x) \mathrm{d} x \text { and } J_{\text {surf }}(\Omega)=\int_{\Gamma} f(x) \mathrm{d} s .
$$

These two functions are shape differentiable at $\Omega$ and

$$
\frac{\partial J_{v o l}}{\partial \Omega}(\Omega)(\mathbf{u})=\int_{\Gamma} f(\mathbf{u . n}) \mathrm{d} s \text { and } \frac{\partial J_{\text {surf }}}{\partial \Omega}(\Omega)(\mathbf{u})=\int_{\Gamma} \mathbf{u . n}\left(\frac{\partial f}{\partial \mathbf{n}}+H f\right) \mathrm{d} s,
$$

for any $\mathbf{u}$ smooth enough, where $H$ is the mean curvature of $\Gamma$ defined by $H=\nabla \cdot \tilde{\mathbf{n}}$ and $\tilde{\mathbf{n}}$ is the local extension of the normal $\mathbf{n}$ near $\Gamma$.

Lemma A.2. (When the integrand depends on the shape). Let us consider a smooth bounded open set $\Omega \in R^{3}$ and $f(\Omega, x)$ a smooth function defined in $R^{3}$ depending also on the domain. Define

$$
J(\Omega)=\int_{\Gamma} f(\Omega) \mathrm{d} s .
$$

This function is shape differentiable at $\Omega$ regarding Fréchet and, for any smooth function $\mathbf{u}$, we have:

$$
\frac{\partial J}{\partial \Omega}(\Omega)(\mathbf{u})=\int_{\Gamma}\left(\frac{\partial f}{\partial \Omega}(\Omega)+\mathbf{u . n}\left(\frac{\partial f(\Omega)}{\partial \mathbf{n}}+H f(\Omega)\right)\right) \mathrm{d} s .
$$

The next part is intended to give a simple derivation of the Murat-Simon expressions: Lemma $A .1$ and Lemma $A .2$, in the two dimensional case.

\section{B. On a simple derivation of the above announced Lemma}

Let us start with a two dimensional problem (the boundary is a line)

$$
J(\Omega)=\int_{\Gamma} f(x) d s
$$

$d s$ is the curvilinear coordinate, and $f$ is a function which does not depend explicitly on the form, but only on $x$ which is a $2 \mathrm{D}$ vector field. Of course $x$ is evaluated at the boundary, since the integral is along the boundary. We have $d s=d \alpha \sqrt{g}$, where $\alpha$ is a parametrization of a curve (for example if $x(\alpha)$ is parametric representation of a curve, then $g=x^{\prime 2}$, where prime denotes differentiation with respect to argument). We can thus write

$$
J(\Omega)=\int_{\Gamma} f(x) \sqrt{g} d \alpha
$$

Since now integration is performed on the parameter $\alpha$ which does not depend on the curve (think of time parametrizing a trajectory!), the variation of $J$ with the shape acquires the simple Euler-Lagrange derivative

$$
R=\frac{\partial(f \sqrt{g})}{\partial x}-\frac{d}{d \alpha} \frac{\partial(f \sqrt{g})}{\partial x^{\prime}}
$$


Expliciting $R$ we have

$$
R=\sqrt{g} \frac{\partial}{\partial x} f-f \frac{d}{d \alpha} \frac{\partial}{\partial x^{\prime}} \sqrt{g}-\frac{d f}{d \alpha} \frac{\partial \sqrt{g}}{\partial x^{\prime}}
$$

We have $\partial \sqrt{g} / \partial x^{\prime}=x^{\prime} / \sqrt{g}, d / d \alpha(\sqrt{g})=x^{\prime} \cdot x^{\prime \prime} / \sqrt{g}$, and $d / d \alpha\left(x^{\prime} / \sqrt{g}\right)=\left[x^{\prime \prime} \sqrt{g}-\right.$ $\left.x^{\prime}\left(x^{\prime} \cdot x^{\prime \prime}\right) / \sqrt{g}\right] / g$. Using the result $r^{\prime}=\sqrt{g} \mathbf{t}$ (coming from the very definition of the tangent vector $\mathbf{t}$ ), and $\mathbf{t}^{\prime}=-H \mathbf{n} \sqrt{g}$, we easily find

$$
R=\sqrt{g}\left[H f \mathbf{n}-\mathbf{t} \frac{\partial f}{\partial s}+\frac{\partial f}{\partial x}\right]
$$

Actually the force is defined as $R / \sqrt{g}$ (see [9]; in fact the above $R$ is derivative with respect to shape at given $\alpha$, while the physical force is to be defined at given $s$, and according to chain rule $\partial / \partial s=\partial /(\sqrt{g} \partial \alpha))$, and we can write finally the variation with respect to the shape

$$
\left[H f \mathbf{n}+\frac{\partial f}{\partial \mathbf{n}} \mathbf{n}\right]
$$

since only the normal enters, this means that only normal motion matters. If $\mathbf{u}$ is displacement, the variation with respect to the form acquires the factor u.n. This is the so-called Murrat-Simon expression (see Lemma A.1). We can reconsider that $f$ depends on shape (actually on $x^{\prime}, x^{\prime \prime}$ etc), we find trivially the generalized expression which contains derivative with respect to form (see Lemma A.2). Extension to 3D is elementary.

\section{Green formula with Surface operators}

The Green formula with Surface operators is demonstrated in this appendix. Let any scalar function $g$ and any vector field $\mathbf{v}$ defined over the closed surface $\Gamma, \mathbf{n}$ is the unit outward normal vector defined as a signed distance function like in the part 2. By (4), we have

$$
\int_{\Gamma} \nabla_{s} g \cdot \mathbf{v} d s=\int_{\Gamma} \nabla g \cdot \mathbf{v} d s-\int_{\Gamma}(\nabla g \cdot \mathbf{n})(\mathbf{v} \cdot \mathbf{n}) d s
$$

By the green formula, using the fact that $\Gamma$ is a closed surface, we have

$$
\int_{\Gamma} \nabla g \cdot \mathbf{v} d s=-\int_{\Gamma} g \nabla \cdot \mathbf{v} d s
$$

Using (5), (20) gives

$$
\int_{\Gamma} \nabla g \cdot \mathbf{v} d s=-\int_{\Gamma} g \nabla_{s} \cdot \mathbf{v} d s-\int_{\Gamma} g(\nabla \mathbf{v} \cdot \mathbf{n}) \cdot \mathbf{n} d s
$$

In order to simplify the second term in the right hand side of (19), we use the green formula

$$
\int_{\Gamma}(\nabla g \cdot \mathbf{n})(\mathbf{v} \cdot \mathbf{n}) d s=-\int_{\Gamma} g \nabla \cdot((\mathbf{v} \cdot \mathbf{n}) \mathbf{n}) d s
$$


We have $\nabla \cdot((\mathbf{v} \cdot \mathbf{n}) \mathbf{n})=\nabla(\mathbf{v} \cdot \mathbf{n}) \cdot \mathbf{n}+(\mathbf{v} \cdot \mathbf{n}) \nabla \cdot \mathbf{n}$, so

$\nabla \cdot((\mathbf{v} \cdot \mathbf{n}) \mathbf{n})=\mathbf{n} \cdot[(\mathbf{n} \cdot \nabla) \mathbf{v}+(\mathbf{v} \cdot \nabla) \mathbf{n}+\mathbf{v} \wedge \operatorname{rot} \mathbf{n}+\mathbf{n} \wedge \operatorname{rot} \mathbf{v}]+(\mathbf{v} \cdot \mathbf{n}) \nabla \cdot \mathbf{n}$

In (23), we can write successively

$$
\begin{gathered}
\mathbf{n .}[(\mathbf{v .} \nabla) \mathbf{n}]=n_{i} v_{j} \partial_{j} n_{i}=\frac{1}{2} v_{j} \partial\left(n_{i}^{2}\right)=\frac{1}{2} v_{j} \partial(1)=0 \\
\mathbf{n .}[(\mathbf{n} . \nabla) \mathbf{v}]=n_{i} n_{j} \partial_{j} v_{i}=n_{j} n_{i} \partial_{j} v_{i}=\mathbf{n} \otimes \mathbf{n}: \nabla \mathbf{v}
\end{gathered}
$$

$\mathbf{n} .[\mathbf{n} \wedge \operatorname{rot} \mathbf{v}]=0$ because $[\mathbf{n} \times \operatorname{rot} \mathbf{v}]$ and $\mathbf{n}$ are orthogonal

$$
\operatorname{rot} \mathbf{n}=\operatorname{rot} \nabla \delta=0
$$

Combining those results with (19)- (21)- (22) and (23), we obtain

$$
\int_{\Gamma} \nabla_{s} g \cdot \mathbf{v} d s+\int_{\Gamma} g \nabla_{s} \cdot \mathbf{v} d s-\int_{\Gamma} g(\nabla \cdot \mathbf{n}) \mathbf{v} \cdot \mathbf{n} d s=0
$$

